\title{
Recalibration of the Antimicrobial MIC
}

\author{
Daniel Amsterdam PhD, DAAMM, FAAAS, FAAMM, FIDSA \\ Professor Emeritus Departments of Microbiology \& Immunology, Medicine and Pathology Jacobs School of Medicine and Biomedical Sciences \\ University at Buffalo, Buffalo, NY.
}

*Corresponding Author: Daniel Amsterdam, Professor Emeritus Departments of Microbiology \& Immunology, Medicine and Pathology Jacobs School of Medicine and Biomedical Sciences

University at Buffalo, Buffalo, NY.

Received date: April 15, 2021; Accepted date: April 27, 2021; Published date: May 06,2021

Citation: D Amsterdam. (2021) Recalibration of the Antimicrobial MIC. Journal of Clinical and Laboratory Research. 2(4) DOI: 10.31579/27680487/021

Copyright: (C2021 Daniel Amsterdam. This is an open-access article distributed under the terms of the Creative Commons Attribution License, which permits unrestricted use, distribution, and reproduction in any medium, provided the original author and source are credited.

In 2009, the World health organization (WHO) referred to the problem of antibiotics and antibiotic resistance stating, "Antibiotic Resistance one of the three greatest threats to human health." In 2019 (i.e., just as the COVID-19 pandemic was evolving), more than 2.8 million antibiotic-resistant infections were identified in the United States, resulting in morethan 35,000 deaths (CDC 2019).

The initial laboratory assay which demonstrated the activity of an antibacterial compound was performed by Alexander Fleming. He showed that an extract from the mold, Penicillium rubens,could inhibit the growth of several species of Gram-positive bacteria - but not Gramnegative bacteria that were cross-streaked on agar against the diffused Penicillium compound.

In the 93 years since his discovery, clinical laboratories have developed antimicrobial testing methods (ASTs) in and on agar and in liquid media using a wide variety of manual and automated tests (Amsterdam 2014). Results of these assays, the interaction of microbe and anti-microbial agents, are interpreted in the form of categorical values: "S" susceptible; "I" intermediate; "R" - resistant or with numerical equivalents that are defined by two major consensus groups - Clinical and Laboratory Standards Institute (CLSI) in the United States and the European Committee on Antimicrobial Susceptibility Testing (EUCAST) in the European Union. For diffusion tests on agar measured in millimeters and the endpoint minimum inhibitory concentration (MIC) determined in broth "breakpoints" are defined, the dividing line between clinical susceptibility (S), intermediate (I) and resistant. These division points represent the underpinning of ASTs. The question posed here is how successful these results are in predicting a positive clinical outcome in patients with infections. When using mortality as an outcome indicator, several studies demonstrated the reduction in mortality when the first antimicrobial agent administered was "susceptible" (Garnacho-Montero et al. 2003; Vallees et al. 2003; Ibrahim et al. 2000).

It is noteworthy and not surprising that most human disease, other than those caused by infectious agents, are treated by medicating the host. In contrast, therapy for infectious diseases attempts to eliminate the pathogen from the host while minimizing adverse sequelae due to host immune responses and drug ie, antimicrobial agent, side effects.

The impetus over the past several years has been to develop conventional ASTs (either on agaror in broth) with truncated incubation intervals i.e., less than 18 hours, so that antimicrobial therapy can be initiated earlier. Molecular arrays i.e., PCR, capable to detect resistance determinants are not readily available in an assay from Gram-negative and Gram-positive microorganisms. In the family Enterobacteriaceae several hundred mechanisms have been reported causing resistance to $\beta$-lactams, cephalosporins, monobactams and/or carbapenems (Leinberger et al. 2010). $\quad \beta$-lactam resistance within the Enterobacteriaceae has been attributed to several mechanisms which include: ESBLs, Amp C; metallo- $\beta$-lactams, and KPCs. The number of genotypically unique ESBLs total more than 200 (Leinberger et al. 2010).

Although genotypic-based methods can fulfill the promise for rapid and accurate detection and/or confirmation of antimicrobial resistance, phenotypic approaches will have an advantageif resistance to the same antimicrobial agent may be caused by several different mechanisms. Whole genome sequencing (WGS) offers the potential to predict the identity of the offending microorganisms and antimicrobial susceptibility i.e., resistance, from a single assay. Genotypic assays possess the capability to detect resistance but not predict susceptibility. The limitationsof genome-based susceptibility testing and predictions need to be reviewed and approved by consensus organizations. In the interim phenotypic testing needs to be maintained.

Irrespective of the two major approaches to AST, phenotypic or genotypic, it is not readily recognized that the path to antimicrobial resistance is tolerance. Antimicrobial resistance represents the ability of a microorganism to grow in an inhibitory concentration, i.e., the MIC; tolerance represents a state of reduced rate of antimicrobial killing (Brauner et al. 2016).

Phenotypically tolerant bacteria are not genetically different from growing, non-tolerant microbes (Girgis et al. 2012). Contemporary clinical microbiology laboratories determine antimicrobial susceptibility/ resistance via phenotypic susceptibility testing that results the MIC. Unfortunately, this assessment fails to detect the prevalence of antimicrobial tolerance as microbial populations abundant in tolerant cells would exhibit the same MIC as isolates consisting only of rapidly growing cells. Methods have been proposed to screen for tolerance. A recent phenotypic method utilizing a modified two-step agar disc diffusion assay has been described (Gefen et al. 2017). In this assay, a nutrient disc is placed in the zone of inhibition 
produced by the antibiotic-impregnated disc. This permits tolerant microbes to grow within that previously defined inhibitory zone.

Although antimicrobial tolerance has been recognized as a risk factor for poor clinical outcome,laboratory tests to identify and characterize tolerance have not been widely adopted as they lack recognized standards. In addition, they have not been approved by consensus organizations. Clearly, the utilization of the MIC as the sole reference to initiate and achieve positive clinical outcome needs to be reevaluated.

\section{References}

1. Amsterdam D. (2014) Susceptibility testing of antimicrobials in liquid media. Chapter 3 in Antibiotics in Laboratory Medicine, $6^{\text {th }}$ Edition. D. Amsterdam, Editor. Wolters Kluwer, Philadelphia

2. Brauner A, Fridman O, Gefen O, Balaban NQ. (2016) Distinguishing between resistance,tolerance and persistence to antibiotic treatment. Nat Rev Microbiol. 14(5):320-330

3. Centers for Disease Control. 2019 Biggest Threats and Data.

4. Garnacho-Montero J, Garcia-Garmendia JL, BarreroAlmodora A, et al. (2003) Impact of adequate empirical antibiotic therapy on the outcome of patients admitted to the intensivecare unit with sepsis. Crit Care Med. 31(12):27422751

5. Gefen O, Chekol B, Strahilevitz J, Balaban NQ. (2017) TDtest: easy detection of bacterialtolerance and persistence in clinical isolates by a modified disk-diffusion assay. Sci Rep. 7:41284.

6. Girgis HS, Harris K, Tavazoie S. ( 2012) Large mutational target size for rapid emergence ofbacterial persistence. Proc Natl Acad Sci USA. 109(31):12740-45

7. Ibrahim EH, Sherman G, Ward S, et al. (2000) The influence of inadequate antimicrobial treatment of bloodstream infections on patient outcomes in the ICU. Chest. 118(1):145155

8. Leinberger DM, Grimm V, Rubtsova M, et al. (2010) Integrated detection of extended- spectrum-beta-lactam resistance by DNA microarray-base genotyping of TEM, SHV and CTX-Mgenes. J Clin Microbiol. 48(2):460-471

9. Valles J, Rello J, Ochagavia A, et al. 2003. Communityacquired bloodstream infection in critically ill adult patients: impact on shock and inappropriate antibiotic therapy on survival.Chest. 123(5):1615-1624 